\title{
Immunocompromised patients with SARS-CoV-2 infection in intensive care units, outcome and mortality
}

\section{Cynthia DENIS ${ }^{1,2 *}$, Hamid MERDJI ${ }^{3}$, Mathieu BALDACINI ${ }^{1}$, Maleka SCHENCK ${ }^{1}$, Thierry ARTZNER ${ }^{1}$, Yoann GRIMAUD ${ }^{1,2}$, Thierry LAVIGNE${ }^{4}$, Ferhat MEZIANI ${ }^{3}$, Vincent CASTELAIN ${ }^{1}$, Raphaël CLERE-JEHL ${ }^{3}$, Francis SCHNEIDER ${ }^{1}$ and Guillaume MOREL $^{1}$}

\author{
${ }^{1}$ Intensive Medicine-Resuscitation Department, Faculty of Medicine, Strasbourg University \\ Hospitals, Hautepierre Hospital, University of Strasbourg, Strasbourg, France \\ ${ }^{2}$ Department of Oncology and Hematology, Institut de Cancérologie Strasbourg. Europe (ICANS), \\ Strasbourg, France \\ ${ }^{3}$ Intensive Medicine-Resuscitation Department, Faculty of Medicine, Strasbourg University \\ Hospitals, New Civil Hospital, University of Strasbourg, Strasbourg, France \\ ${ }^{4}$ Hospital Hygiene Service, Public Health Pole, Strasbourg University Hospitals, France
}

\section{Abstract}

Background: The new severe acute respiratory syndrome coronavirus 2 (SARS-CoV-2) outbreak severely hit Northeastern France from March to May 2020. The massive arrival of SARS-CoV-2 positive patients in the intensive care units (ICU) raised the question of how immunocompromised patients would be affected. Therefore, we analyzed the clinical, biological and radiological features of 24 immunocompromised ICU patients with severe SAR-CoV-2 infection.

Results: The mortality rate was significantly higher for immunocompromised patients compared with other patients $(41.7 \%$ versus $27.3 \%$, respectively, $p=0.021)$. Mortality occurred in the first 2 weeks of intensive care, highlighting the possible interest in prolonged full-code managnement of these patients. Finally, patients with lymphoid malignancies appeared to be particularly affected, mostly with monoclonal gamma-pathology.

Conclusion: Mortality rate of SARS-CoV-2 acute respiratory syndrome in immunocompromised patient is high. No treatment was associated with survival improvement. Prolonged full-code management is required for these patients.

\section{Background}

The severe acute respiratory syndrome coronavirus 2 (SARS-CoV-2) epidemic affected Northeastern France early on, with an uncontrolled cluster arising near our hospital in March 2020 [1,2]. Our Intensive Care Units (ICU) were confronted with a massive number of patients in need of critical care. In our ICU, critically ill and immunocompromised patients (ICP) have been treated for decades with the same consideration as other critically ill patients. However, with the massive arrival of patients with severe SARS-CoV-2 infection, we feared an overwhelming influx of infected immunocompromised patients. We were concerned that these patients would be

\section{More Information}

*Address for Correspondence: Dr. Cynthia Denis, Intensive Medicine,

Resuscitation, Hautepierre Hospital, Strasbourg University Hospitals, France, Tel: +33 3868719; Email: c.denis@icans.eu

Submitted: August 10, 2021

Approved: August 16, 2021

Published: August 17, 2021

How to cite this article: DENIS C, MERDJI H, BALDACINI M, SCHENCK M, ARTZNER T, et al. Immunocompromised patients with SARSCoV-2 infection in intensive care units, outcome and mortality. J Clin Intensive Care Med. 2021; 6: 021-025.

\section{DOI: 10.29328/journal.jcicm.1001036}

ORCiD: orcid.org/0000-0002-6508-7253

Copyright: (c) 2021 DENIS C, et al. This is an open access article distributed under the Creative Commons Attribution License, which permits unrestricted use, distribution, and reproduction in any medium, provided the original work is properly cited.

Keywords: SARS-CoV-2; Critical care; Immunocompromised

Check for updates

OPEN ACCESS 
treatment with immune suppressive drugs for any medical condition were considered to be immunocompromised (ICP). Information on demographics, medical history, SARS-CoV-2 infection, biological parameters, treatments and outcome were collected. Simplified acute physiology score (SAPSII) [3] and sequential organ failure assessment score (SOFA) [4] were calculated. Radiological severity was assessed by an independent radiologist, according to the Fleischner Society's consensus statement [5] as mild, moderate-to-severe or critical lesions. A SARS-CoV-2 swab sample was performed at admission and then weekly during the ICU stay and analyzed using Real Time Polymerase Chain Reaction (RT-PCR). All patients received a notice of information and this study was approved by the local institutional review board. The patients were not participating to any other trial concerning SARSCoV-2 during the study period.
Continuous variables are expressed as medians with interquartile ranges (IQR). Categorical variables are described as counts with percentages. Comparisons were performed using Fisher's exact test, Wilcoxon rank-sum test and chi-square test of independence. Overall ICU survival was defined as the time between ICU admission and the date of death or last follow-up. Survival curves were established with a Kaplan-Meier's method and the difference between immunocompetent and immunocompromised patients was analyzed by a log-rank test. Analysis were performed on $\mathrm{R}$ studio software (4.0.3 version).

\section{Results}

Between March to May 2020, 233 patients were admitted to the two medical ICUs for severe SARS-CoV-2 infection, among which 24 (10.3\%) were ICP. Clinical and biological

Table 1: Characteristics of immunocompromised patients and of their ICU-stay according to survival.

\begin{tabular}{|c|c|c|c|c|c|}
\hline Characteristics & $\mathbf{N}$ & Overall, $n=24$ & Survivors, $n=14$ & Deceased, $n=10$ & $p$ - value \\
\hline Male & 24 & $13(54 \%)$ & $7(50 \%)$ & $6(60 \%)$ & 0.70 \\
\hline Age & 24 & $68(57-71)$ & $64(47-71)$ & $68(64-70)$ & 0.32 \\
\hline$E C O G>2$ & 24 & $7(29 \%)$ & $4(29 \%)$ & $3(30 \%)$ & $>0.99$ \\
\hline Body mass index $\left(\mathrm{kg} / \mathrm{m}^{2}\right)$ & 24 & $27.5(26.0-32.0)$ & $28.0(26.0-31.8)$ & $27.5(24.5-34.0)$ & $>0.99$ \\
\hline Immunodeficiency causes & 24 & & & & \\
\hline Solid organ cancers ${ }^{a}$ & & $12(50 \%)$ & $8(57 \%)$ & $4(40 \%)$ & 0.68 \\
\hline Hematologic maligncies $^{b}$ & & $8(33 \%)$ & $3(21 \%)$ & $5(50 \%)$ & 0.20 \\
\hline Solid organ transplant recipients & & $4(17 \%)$ & $3(21 \%)$ & $1(10 \%)$ & 0.61 \\
\hline \multicolumn{6}{|l|}{ Medical comorbidities } \\
\hline Cardiovascular comorbidity & 24 & $12(50 \%)$ & $7(50 \%)$ & $5(50 \%)$ & $>0.99$ \\
\hline Diabetes mellitus & 24 & $6(25 \%)$ & $2(14 \%)$ & $4(40 \%)$ & 0.19 \\
\hline Chronic kidney failure & 24 & $6(25 \%)$ & $3(21 \%)$ & $3(30 \%)$ & 0.67 \\
\hline \multicolumn{6}{|l|}{ Prognostic scores at admission } \\
\hline SOFA & 24 & $7.0(4.8-11.0)$ & $5.0(4.0-7.0)$ & $10.5(8.0-12.0)$ & 0.01 \\
\hline SAPS II & 24 & $50(39-73)$ & $40(33-47)$ & $70(63-75)$ & 0.01 \\
\hline \multicolumn{6}{|l|}{ Organ support in ICU } \\
\hline Mechanical ventilation & 24 & $22(92 \%)$ & $12(86 \%)$ & $10(100 \%)$ & 0.49 \\
\hline Optiflow ${ }^{\circledR}$ ventilation before intubation & 24 & $7(29 \%)$ & $2(14 \%)$ & $5(50 \%)$ & 0.09 \\
\hline Mechanical ventilation: days & 22 & $9(6-15)$ & $9(7-20)$ & $9(4-10)$ & 0.19 \\
\hline $\mathrm{PaO}_{2} / \mathrm{FiO}_{2}$ & 23 & $97(64-114)$ & $105(82-117)$ & $74(53-106)$ & 0.17 \\
\hline Prone positioning & 24 & $10(42 \%)$ & $5(36 \%)$ & $5(50 \%)$ & 0.68 \\
\hline Neuromuscular blockade & 24 & $15(62 \%)$ & $7(50 \%)$ & $8(80 \%)$ & 0.21 \\
\hline Inhaled nitric oxide & 24 & $8(33 \%)$ & $5(36 \%)$ & $3(30 \%)$ & $>0.99$ \\
\hline ECMO & 24 & $1(4.2 \%)$ & $1(7.1 \%)$ & $0(0 \%)$ & $>0.99$ \\
\hline Renal-replacement therapy & 24 & $8(33 \%)$ & $4(29 \%)$ & $4(40 \%)$ & 0.67 \\
\hline Vasopressors & 24 & $19(79 \%)$ & $10(71 \%)$ & $9(90 \%)$ & 0.36 \\
\hline \multicolumn{6}{|c|}{ Biological parameters at ICU admission } \\
\hline Leukocytes $\left(.10^{9} / \mathrm{L}\right)$ & 24 & $6.0(5.2-8.3)$ & $6.0(5.2-7.1)$ & $6.6(5.4-8.7)$ & $>0.99$ \\
\hline Lymphocytes (.10\%/L) & 24 & $0.5(0.2-0.8)$ & $0.4(0.2-0.7)$ & $0.6(0.3-2.1)$ & 0.17 \\
\hline Platelets $\left(.10^{9} / \mathrm{L}\right)$ & 24 & $158(75-245)$ & $196(57-260)$ & $144(88-182)$ & 0.63 \\
\hline Creatinin $(\mu \mathrm{mol} / \mathrm{L})$ & 24 & $73(63-128)$ & $81(70-148)$ & $69(61-102)$ & 0.84 \\
\hline C-Reactive Protein (mg/L) & 24 & $114(49-202)$ & $128(48-224)$ & $114(60-170)$ & 0.71 \\
\hline D-dimers $(\mu \mathrm{g} / \mathrm{L})$ & 24 & $2,605(1,160-4,055)$ & $2,475(1,362-4,540)$ & $2,895(1,160-3,662)$ & 0.79 \\
\hline Positive lupus anticoagulant & 14 & $11(79 \%)$ & $8(80 \%)$ & $3(75 \%)$ & $>0.99$ \\
\hline \multicolumn{6}{|l|}{ CT-Scan features } \\
\hline Mild & 17 & $3(18 \%)$ & $2(17 \%)$ & $1(20 \%)$ & $>0.99$ \\
\hline Moderate to severe & 17 & $10(59 \%)$ & $8(67 \%)$ & $2(40 \%)$ & 0.59 \\
\hline Critical & 17 & $4(24 \%)$ & $2(17 \%)$ & $2(40 \%)$ & 0.54 \\
\hline
\end{tabular}

a: Cancer patients : renal clear cell carcinoma (2); pulmonary adenocarcinoma with ALK mutation (1); rectal adenocarcinoma (1); osteosarcoma (1); thymoma (1); Ewing sarcoma (1); prostate adenocarcinoma (1); vesical carcinoma (1); urothelial carcinoma (1); thyroid carcinoma (1); endometrial carcinoma (1); pancreatic adenocarcinoma (1). b: Hematologic patients : multiple myeloma (2); marginal zone lymphoma (2); mantle cell lymphoma (1); IgA kappa type lympho-plasmacytic lymphoma (1); chronic myelomonocytic leukemia (1); acute pro-myelocytic leuke@mia (1). 
characteristics of ICP patients with SARS- CoV-2 infection are reported in Table 1 . Twelve (50\%) patients had a history of active cancer in the last five years (see histological types Table 1). Three of the oncology patients also had a history of hematological malignancies (lymphoid malignancies). Eight patients had a history of hematological malignancies, consisting of lymphoproliferative disorders in 6 of the 8 cases (75\%). Within 3 months previous to admission, 2 patients had undergone autologous stem-cell transplantation. Among the onco-hematological patients, 12 (50\%) received chemotherapy in the previous 3 months. The four remaining patients were solid organ transplant recipients, who had received kidney $(n=3)$ and liver $(n=1)$ transplants. No HIV patients were hospitalized during this period. Interestingly, six patients (25\%) displayed monoclonal gamma-pathology. The median time between the first symptoms and ICU admission was $9(6.2-11)$ days.

The severity of the acute respiratory distress syndromes was illustrated by the presence of moderate to critical radiological infiltrates in most of the patients $(n=14,82 \%)$, low $\mathrm{PaO}_{2} / \mathrm{FiO}_{2}$ ratios, especially in the deceased group, high ICU prognostic scores and the need for invasive mechanical ventilation in all but 2 patients. Of note, non-invasiveventilation was frequently insufficient to treat acute respiratory failure in our ICP patients. One patient successfully underwent ECMO for 32 days.

For specific treatment of SARS-CoV-2 infection, 6 (25\%) patients received Hydroxychloroquine, 6 (25\%) LopinavirRitonavir, 9 (38\%) Azithromycin, 1 (4\%) Interferon-beta, and 7 (29\%) steroids (2 mg/kg Prednisolone). None of these treatments showed a statistical difference on survival.

Eleven patients (79\%) had a positive lupus anticoagulant. All but one received anticoagulant therapy, 11 (48\%) of them receiving therapeutic anticoagulation. We observed venous thromboembolism events in 3 patients and one patient, with newly diagnosed promyelocytic leukemia, died from a carotid artery occlusion.

Healthcare-associated infections developed in 9 (37\%) patients, mostly ventilator-associated pneumonia (78\%). Only one patient had airway colonization by Aspergillus fumigatus, without proof for invasive infection. Interestingly $67 \%$ and $50 \%$ of the patients still had a positive SARS-CoV-2 PCR result from the SARS-CoV-2 swab sample at days 21 and 28 of ICU stay, respectively, suggesting prolonged viral carriage in ICP patients.

The median length of stay in the ICU was 9 days (IQR 7-26) for the patients who recovered and 4 days (IQR 4-12) for the deceased patients, respectively $(p=0.4)$. Figure 1 shows the survival curve of all SARS-CoV-2 infected patients, ICP or not. At day 28, the ICP patients had a significantly lower survival rate compared with other patients (41.7\% versus $27.3 \%$, $p=0.021$ ). The causes of death were respiratory failure

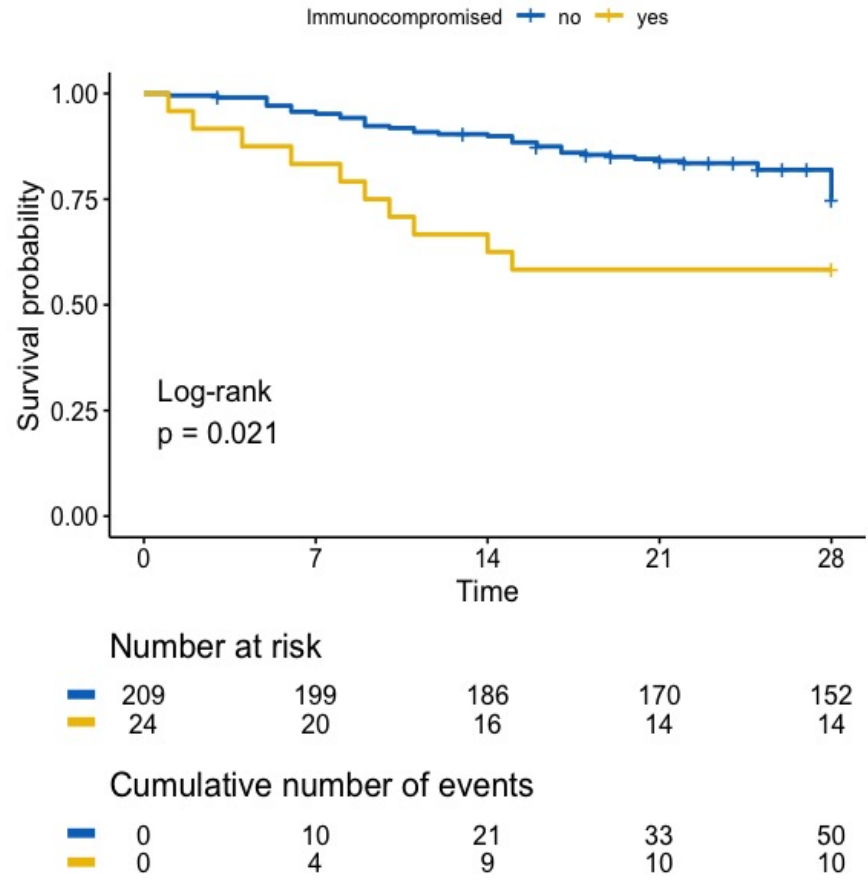

Figure 1: Overall survival of patients with SARS-CoV-2 infection. Mortality of ICP with SARS-CoV-2 infection is significantly higher than mortality of immunocompetent patients.

(50\%), sepsis (40\%) and thrombosis (10\%). No detectable factors were significantly different between SARS-CoV-2 ICP patients who recovered and those who did not, with the exception of classic scores (SAPSII, SOFA), which were higher in the deceased group (Table 1).

\section{Discussion}

Here we describe the global characteristics, severity and outcome of immunocompromised patients with severe SARS-CoV-2 infection admitted in two medical intensive care units. These patients represented $10 \%$ of the total number of patients admitted in our ICU during the first wave of the SARSCoV-2 outbreak, an equal proportion to what was described by the COVID-ICU Group [1]. The 28-day mortality rate in the intensive care unit observed for immunocompetent patients with SARS-CoV-2 infection was a bit lower to that reported in the literature for ICU patients with ARDS due to SARSCoV-2 infection [1,6], in comparison with immunocompetent patients, the mortality of immunocompromised patients was significantly higher (Figure 1). This was a striking difference from what is expected for immunocompromised patients in ICU, whose survival rate is nowadays comparable to that of immunocompetent patients, particularly in the context of respiratory infections, such as influenza $[7,8]$. In their recent review, Fung, et al. [9] also point out this outstanding mortality of immunocompromised patients with SARS-CoV-2 infection, and being immunocompromised is associated with 90-day mortality in the COVID-ICU group publication [1].

In addition, patients with lymphoid malignancies, especially 
those with monoclonal gamma-pathy, seem to be particularly susceptible to SARS-CoV-2 infection, a particularity also highlighted by Malard, et al. [10]. This observation once again distinguishes the SARS-COV-2 infection since patients with lymphoid malignancies are not hematological patients usually admitted to ICU [8].

It is also important to notice that despite a clear severity of SARS-CoV-2 infection in immunocompromised patients, accompanied by high mortality in the early days, no ICP patient died beyond day 14 in ICU, highlighting a possible interest in prolonged full-code management for these patients in the ICU.

The clinical and biological characteristics of immunocompromised patients were comparable to the overall characteristics of all patients with SARS-CoV-2 infection $[1,11]$. The immunocompromised patients also developed ventilatoracquired pneumonia which negatively affects their prognosis. However, although at high theoretical risk, our patients did not undergo invasive fungal infections, in contrast with what has been reported during influenza infections [8] or in other publications on SARS-CoV-2 [12]. Thromboses were observed in immunocompromised patients requiring the use of curative anticoagulation $[13,14]$, which is difficult to implement in these patients who are readily thrombocytopenic. In addition, no treatment (in particular no anti-viral therapy), showed any significant impact on the mortality rate, in accordance with recent publications $[9,15]$.

One final striking result is the prolonged viral excretion. Indeed, one month after the onset of symptoms, more than half of patients had still a detectable viral load in the respiratory fluids, raising the issue of maintaining isolation measures.

Although the number of patients in our study is low, but in full accordance with the expected rate of admissions of such patients in our facility over a two-month period, we did not identify significant differences between immunocompromised and -competent groups except for mortality rates. This study nevertheless provides a detailed description of the characteristics of ICP patients, and the management challenges of these patients admitted to intensive care with severe SARSCoV-2 infection, from a single center point of view.

\section{Conclusion}

The population of immunocompromised patients, especially patients with lymphoid malignancies like monoclonal gammopathy, seem to be those most affected by SARS- CoV-2 infection, with an unusually high mortality rate. Special attention should be paid to these patients for whom prolonged resuscitation seems required.

\section{Declarations}

Ethics approval statement: All patients received an information notice and the study was approved by the local institutional review board.
Consent for publication: The authors accepted the manuscript for publication.

Authors' contributions: All authors contributed to data collection. CD, HM, MB, MS, TA, TL, RCJ, YG and GM analyzed and interpreted the patient data. CD, TA, FM, VC, FS and GM were major contributors in writing the manuscript. All authors read and approved the final manuscript.

\section{Acknowledgement}

We thank Susan Chan for editing.

\section{References}

1. COVID-ICU Group on behalf of the REVA Network and the COVID-ICU Investigators. Clinical characteristics and day-90 outcomes of 4244 critically ill adults with COVID-19: a prospective cohort study. Intensive Care Med. 2021; 47: 60-73.

PubMed: https://pubmed.ncbi.nlm.nih.gov/33211135/

2. Kuteifan $\mathrm{K}$, Pasquier $\mathrm{P}$, Meyer $\mathrm{C}$, Escarment $\mathrm{J}$, Theissen $\mathrm{O}$. The outbreak of COVID-19 in Mulhouse: Hospital crisis management and deployment of military hospital during the outbreak of COVID-19 in Mulhouse, France. Ann Intensive Care. 2020; 10: 59.

PubMed: https://pubmed.ncbi.nlm.nih.gov/32430597/

3. Le Gall JR, Lemeshow S, Saulnier F. A new Simplified Acute Physiology Score (SAPS II) based on a European/North American multicenter study. JAMA. 1993; 270: 2957-2963.

PubMed: https://pubmed.ncbi.nlm.nih.gov/8254858/

4. Moreno R, Vincent JL, Matos R, Mendonça A, Cantraine F, et al. The use of maximum SOFA score to quantify organ dysfunction/failure in intensive care. Results of a prospective, multicentre study. Working Group on Sepsis related Problems of the ESICM. Intensive Care Med. 1999; 25: 686-696.

PubMed: https://pubmed.ncbi.nlm.nih.gov/10470572/

5. Rubin GD, Ryerson CJ, Haramati LB, Sverzellati N, Kanne JP, et al The Role of Chest Imaging in Patient Management during the COVID-19 Pandemic: A Multinational Consensus Statement from the Fleischner Society. Radiology. 2020; 158: 106-116.

6. Auld SC, Caridi-Scheible M, Robichaux C, Coopersmith CM, Murphy DJ, et al. Declines in Mortality Over Time for Critically III Adults with Coronavirus Disease 2019. Crit Care Med. 2020; 48: e1382-e1384. PubMed: https://pubmed.ncbi.nlm.nih.gov/32991356/

7. Martin-Loeches I, Lemiale V, Geoghegan P, McMahon MA, Pickkers P, et al. Influenza and associated co-infections in critically ill immunosuppressed patients. Crit Care. 2019; 23: 152. PubMed: https://pubmed.ncbi.nlm.nih.gov/31046842/

8. Azoulay E, Mokart D, Kouatchet A, Demoule A, Lemiale V. Acute respiratory failure in immunocompromised adults. Lancet Respir Med. 2019; 7: 173-186.

PubMed: https://pubmed.ncbi.nlm.nih.gov/30529232/

9. Fung M, Babik JM. COVID-19 in immunocompromised hosts: What We Know So Far. Clin Infect Dis. 2021; 72: 340-350. PubMed: https://pubmed.ncbi.nlm.nih.gov/33501974/

10. Malard F, Genthon A, Brissot E, van de Wyngaert Z, Marjanovic Z, et al COVID-19 outcomes in patients with hematologic disease. Bone Marrow Transplant. 2020; 55: 2180-2184.

PubMed: https://pubmed.ncbi.nlm.nih.gov/32376969/

11. Guan WJ, Ni ZY, Hu Y, et al. Clinical Characteristics of Coronavirus Disease 2019 in China. N Engl J Med. 2020; 382: 1708-1720. 
12. Bartoletti $M$, Pascale $R$, Cricca $M$, Rinaldi $M$, Maccaro $A$, et al. Epidemiology of invasive pulmonary aspergillosis among COVID-19 intubated patients: a prospective study. Clin Infect Dis. 2020; ciaa1065. PubMed: https://pubmed.ncbi.nlm.nih.gov/32719848/

13. Helms J, Tacquard C, Severac F, Leonard-Lorant I, Ohana M, et al. High risk of thrombosis in patients with severe SARS-CoV-2 infection: a multicenter prospective cohort study. Intensive Care Med. 2020; 46 : 1089-1098.

PubMed: https://pubmed.ncbi.nlm.nih.gov/32367170/
14. Baldacini M, Pop R, Sattler L, Mauvieux L, Bilger K, et al. Concomitant haemorrhagic syndrome and recurrent extensive arterial thrombosis in a patient with COVID-19 and acute promyelocytic leukaemia. $\mathrm{Br} \mathrm{J}$ Haematol. 2020; 189: 1054-1056.

PubMed: https://pubmed.ncbi.nlm.nih.gov/32369614/

15. Consortium WHOST, Pan H, Peto R, Henao-Restrepo AM, Preziosi MP, et al. Repurposed Antiviral Drugs for Covid-19 - Interim WHO Solidarity Trial Results. N Engl J Med. 2021; 384: 497-511.

PubMed: https://pubmed.ncbi.nlm.nih.gov/33264556/ 